\title{
CROSSING-CHANGEABLE BRAIDS FROM CHROMATIC CONFIGURATION SPACES
}

\author{
HAO LI AND ZHI LÜ
}

\begin{abstract}
Motivated by the work in [15], this paper deals with the theory of the braids from chromatic configuration spaces. This kind of braids possess the property that some strings of each braid may intersect together and can also be untangled, so they are quite different from the ordinary braids in the sense of Artin. This enriches and extends the theory of ordinary braids.
\end{abstract}

\section{INTRODUCTION}

Generally, the mathematical study of braids can be traced back to the seminar work of Artin in [1, 2] around the first half of the last century. The theory of braids studies the concept of braids and the presentations of braid groups as well as various generalizations arising from various branches of the mathematics. For example, braid groups can be interpreted as the fundamental groups of certain (unordered) configuration spaces by Fox and Neuwirth [13]. Brieskorn [8, 9] extended the notion of the braid group to Artin groups or the generalized braid groups by associating to all finite Coxeter groups. So far, there have been many interesting and strong links between the theory of braids and other various theories and areas, such as knot theory, group theory, algebraic geometry, mathematical physics and so on (e.g., see [3, 4, 6, 7, 10, 11, 14]).

As pointed out in [15], the theory of ordinary braids has two basic theoretical features: One is that each braid group is realized as the fundamental group of the orbit space of a geometric object with free action of a group; the other is that each braid group uniquely corresponds to a short exact sequence induced by the geometric object with free action. In [15], the theory of orbit braids has been established by making use of the construction of orbit configuration spaces (which can be understood as a generalization of classical configuration spaces), and generally it does not possess the above basic theoretical features. The theory of the orbit braids from orbit configuration spaces provides us with much more insights. Indeed, an orbit braid group can be large enough to contain various different braid groups as subgroups, but it can still be described in terms of homotopy (i.e., the so-called extended fundamental group defined in [15]).

Motivated by the work in [15], in this paper we consider the braids from chromatic configuration spaces (which can be regarded as another generalization of classical configuration spaces). This kind of braids are quite different from the ordinary braids in the sense of Artin. Actually some strings of such a braid may intersect together but we can untangle them by the construction of chromatic configuration spaces. Of course,

Key words and phrases. Crossing-changable braid, chromatic configuration space, extended fundamental group.

Partially supported by the NSFC grants (No. 11971112 and 11431009). 
the groups formed by such braids don't possess the above basic theoretical features of the theory of ordinary braids yet. The purpose of this paper is to deal with the theory of such braids from chromatic configuration spaces.

Let $\Gamma$ be a simple graph without loops on the vertex set $[n]=\{1, \ldots, n\}$. By $E(\Gamma)$ we denote the set of edges of $\Gamma$, and by $\overline{i j}$ we denote the edge adjacent to two vertices $i, j \in[n]$. Then the chromatic configuration space of a topological space $X$ over $\Gamma$ is defined by

$$
F(X, \Gamma)=\left\{\left(x_{1}, \ldots, x_{n}\right) \in X^{\times n} \mid x_{j} \neq x_{k} \text { for } \overline{j k} \in E(\Gamma)\right\} \text { \}. }
$$

If we regard each point in $X$ as a color, then $F(X, \Gamma)$ exactly consists of all colorings by using all colors of $X$ to color vertices of $\Gamma$ in such a way that adjacent vertices always have different colors. So this is reason why we call $F(X, \Gamma)$ the chromatic configuration space of $X$ over $\Gamma$. The definition of $F(X, \Gamma)$ first appeared in the work of Eastwood and Huggett [12], where $F(X, \Gamma)$ was called the generalized configuration space therein. Clearly, if $\Gamma$ is a complete graph, then $F(X, \Gamma)$ is just the classical configuration space $F(X, n)$, so the chromatic configuration spaces enrich the world of classical configuration spaces, and in particular, they also bring in much information of graphs to configuration spaces.

The chromatic configuration space $F(X, \Gamma)$ possesses the distinctive properties in its own way. First, the symmetric group $\Sigma_{n}$ does not act on $F(X, \Gamma)$ very well, but its role will be replaced by $\operatorname{Aut}(\Gamma)$, the automorphism group of the graph $\Gamma$. However, the canonical action of $\operatorname{Aut}(\Gamma)$ on $F(X, \Gamma)$ is not free in general. This results in the failure of existence of fibre map, thus it is not surprising that the braid group from the chromatic configuration space that we will consider can not be realized as the fundamental group of some topological space. Second, $F(X, \Gamma)$ relies on the structure of $\Gamma$ heavily. It is well-known that the structure of graphs is complicated, especially, the exact status of Aut $(\Gamma)$ is still unknown [5]. These two factors lead to difficulties when considering $F(X, \Gamma)$.

Following the idea in [15], the concept of braid groups can actually be generalised to various configuration spaces. Now let us deal with the theory of the braids from the chromatic configuration spaces. Assume that $X$ is a connected topological manifold of dimension at least two. Choose a base point $\mathbf{x}$ in $F(X, \Gamma)$, which is of free orbit type under the action of $\operatorname{Aut}(\Gamma)$. Then we will perform our work as follows:

(1) Use the paths $\alpha$ with starting point $\mathbf{x}$ and ending point in the orbit $\operatorname{Aut}(\Gamma)(\mathbf{x})$ at $\mathbf{x}$ in $F(X, \Gamma)$ to construct the geometric braids $c(\alpha)$ with $n$ strings in $X \times I$. Such a geometric braid $c(\alpha)$ is quite different from one in the sense of Artin. Actually, there may be intersection points between different strings in $c(\alpha)$. Intersection or non-intersection of different strings in $c(\alpha)$ depend upon the structure of $\Gamma$. However, any intersection point appearing in different strings can be untangled by doing a small deformation on the path $\alpha$ in $F(X, \Gamma)$. This means that if

\footnotetext{
${ }^{*}$ If $X$ admits an effective action of a group $G$, then we can even define the chromatic orbit configuration space

$$
F_{G}(X, \Gamma)=\left\{\left(x_{1}, \ldots, x_{n}\right) \in X^{\times n} \mid G\left(x_{j}\right) \neq G\left(x_{k}\right) \text { for } \overline{j k} \in E(\Gamma)\right\}
$$

where $G(x)$ denotes the orbit at $x \in X$. Certainly we can also consider the braids from the chromatic orbit configuration spaces, but more details will be involved. Here for our purpose we mainly pay our attention on dealing with the theory of the braids from chromatic configuration spaces.
} 
two strings can intersect, then any crossing happening between them can be changed via the intersection point. Thus, such a geometric braid $c(\alpha)$ is also called a crossing-changeable braid.

(2) Define an equivalence relation among crossing-changeable braids $c(\alpha)$ such that the relation agrees with the homotopy relation (relative to $\partial I$ ) among the paths $\alpha$. Furthermore, all equivalence classes of crossing-changeable braids form a group, denoted by $B(X, \Gamma)$, which is called the crossing-changeable braid group. At the same time, all homotopy classes (relative to $\partial I$ ) of the paths with starting point $\mathbf{x}$ and ending point in $\operatorname{Aut}(\Gamma)(\mathbf{x})$ in $F(X, \Gamma)$ can also form a group by equipping with an operation, denoted by $\pi_{1}^{E}(F(X, \Gamma), \mathbf{x}, \operatorname{Aut}(\Gamma)(\mathbf{x}))$, which is called the extended fundamental group It $\mathrm{F}(X, \Gamma)$, defined in [15].

The following theorem tells us that the crossing-changeable braid group $B(X, \Gamma)$ can be described in terms of the extended fundamental group $\pi_{1}^{E}(F(X, \Gamma), \mathbf{x}, \operatorname{Aut}(\Gamma)(\mathbf{x}))$ homotopically.

Theorem 1.1. The crossing-changeable braid group $B(X, \Gamma)$ is isomorphic to the extended fundamental group $\pi_{1}^{E}(F(X, \Gamma), \mathbf{x}, \operatorname{Aut}(\Gamma)(\mathbf{x}))$.

Compared with ordinary braid groups, the structure of $B(X, \Gamma)$ seems to be more complicated. As is known, a braid in the ordinary braid group $B(X, n)$ can always be decomposed at each crossing of $n$ strings, so what we need to do is to consider the braids with only one crossing and then to find out the relations among them. However, for a crossing-changeable braid in $B(X, \Gamma)$, doing the same decomposition does not work very well. In fact, since once we decompose it at each crossing, the induced braids may not lie in $B(X, \Gamma)$. This is the key point where difficulty lies in.

Like the theory of ordinary braid groups, $B(X, \Gamma)$ contains a subgroup $P(X, \Gamma)$, called the pure crossing-changeable braid group, which is exactly isomorphic to the fundamental group $\pi_{1}(F(X, \Gamma), \mathbf{x})$. On the other hand, each class of $B(X, \Gamma)$ determines a unique element in $\operatorname{Aut}(\Gamma)$. This leads us to obtain a short exact sequence.

Theorem 1.2. There is a short exact sequence

$$
1 \longrightarrow P(X, \Gamma) \longrightarrow B(X, \Gamma) \longrightarrow \operatorname{Aut}(\Gamma) \longrightarrow 1
$$

Using the inclusion $i: F(X, n) \hookrightarrow F(X, \Gamma)$, we also study the relation between the braid groups or the extended fundamental groups of $F(X, n)$ and $F(X, \Gamma)$, see Proposition 2.2.

Finally we focus on the case of $X=\mathbb{C}$. By the presentation of the classical pure braid group, we can give a presentation of $P(\mathbb{C}, \Gamma)$ for any finite simple graph $\Gamma$ (see Theorem 3.3). However, the determination of $B(\mathbb{C}, \Gamma)$ is not an easy task since the group structure of $\operatorname{Aut}(\Gamma)$ is still unknown in general. Making use of the group extension theory, we obtain an explicit presentation of $B\left(\mathbb{C}, C_{n}\right)$ where $C_{n}$ is the cycle graph.

\footnotetext{
${ }^{\dagger}$ As Golasiński Marek told us after this paper was submitted, the extended fundamental group is actually the fundamental group of a transformation group in the sense of Rhodes in [21]. In addition, as noted by Looijenga in [16], such group is also called an equivariant fundamental group, which can be regarded as an orbifold fundamental group. To be compatible with [15], here we still adopt the notation used in [15].
} 
The paper is organized as follows. Section 2 is the main part of this paper, where we will discuss how to define a crossing-changeable braid and the equivalence relation among crossing-changeable braids. Then we construct the crossing-changeable braid group and give it a homotopy description. Furthermore, we establish a short exact sequence about $B(X, \Gamma)$ and $P(X, \Gamma)$. We also discuss the connection between the braid groups and the extended fundamental groups of $F(X, n)$ and $F(X, \Gamma)$. In Section 3, we pay our attention to the case $X=\mathbb{C}$. We calculate $P(\mathbb{C}, \Gamma)$ for arbitrary finite simple graph $\Gamma$, and $B\left(\mathbb{C}, C_{n}\right)$.

\section{CROSSING-CHANGEABLE BRAIDS}

Given a topological space $X$ and a simple graph $\Gamma$ without loops on the vertex set $[n]=\{1, \ldots, n\}$. Then we have the chromatic configuration space of $X$ over $\Gamma$ defined by

$$
F(X, \Gamma)=\left\{\left(x_{1}, \ldots, x_{n}\right) \in X^{\times n} \mid x_{j} \neq x_{k} \quad \text { for } \quad \overline{j k} \in E(\Gamma)\right\}
$$

with subspace topology. In the case when $\Gamma$ is a complete graph, then $F(X, \Gamma)$ is the classical configuration space. However, $F(X, n)$ is always a subspace of $F(X, \Gamma)$ in general.

Let $\operatorname{Aut}(\Gamma)$ denote the automorphism group of $\Gamma$

$$
\operatorname{Aut}(\Gamma)=\left\{\sigma \in \Sigma_{n} \mid \overline{\sigma(j) \sigma(k)} \in E(\Gamma) \Longleftrightarrow \overline{j k} \in E(\Gamma)\right\}
$$

which is a subgroup of the symmetric group $\Sigma_{n}$. It is easy to see that the chromatic configuration space $F(X, \Gamma)$ admits a natural action of Aut $(\Gamma)$, defined by

$$
\left(\sigma,\left(x_{1}, \ldots, x_{n}\right)\right) \longmapsto\left(x_{\sigma(1)}, \ldots, x_{\sigma(n)}\right) .
$$

However, this action on $\operatorname{Aut}(\Gamma)$ on $F(X, \Gamma)$ is generally non-free. Of course, when $\Gamma$ is a complete graph, $\operatorname{Aut}(\Gamma)$ just becomes the symmetric group $\Sigma_{n}$, so that the natural action of $\Sigma_{n}$ on $F(X, n)$ is free.

In the following, we shall pay attentions to the case in which $X$ is a connected topological manifold of dimension greater than one. Then it is easy to see that $F(X, \Gamma)$ is connected.

2.1. Definition and equivalence relation of crossing-changeable braids. Given a path $\alpha=\left(\alpha_{1}, \ldots, \alpha_{n}\right): I \longrightarrow F(X, \Gamma)$, where $\alpha_{i}: I \longrightarrow X$ is the $i$-th coordinate of $\alpha$. Then $\alpha$ uniquely determines a configuration $c(\alpha)=\left\{c\left(\alpha_{1}\right), \ldots, c\left(\alpha_{n}\right)\right\}$ of $n$ strings in $X \times I$, where $c\left(\alpha_{i}\right)=\left\{\left(\alpha_{i}(s), s\right) \mid s \in I\right\}$. By the construction of $F(X, \Gamma)$, it is easy to see that two strings $c\left(\alpha_{j}\right)$ and $c\left(\alpha_{k}\right)$ may intersect if $\overline{j k} \notin E(\Gamma)$. In addition, it should also be emphasized that all $n$ strings in $c(\alpha)$ are unordered in $X \times I$.

Now let us choose a base point $\mathbf{x}=\left(x_{1}, \ldots, x_{n}\right)$ in $F(X, \Gamma)$ such that $x_{i}$ 's are pairwisely distinct in $X$, so $\mathbf{x}$ is of free orbit type under the action of Aut $(\Gamma)$. Given a $\sigma \in \operatorname{Aut}(\Gamma)$, we denote $\left(x_{\sigma(1)}, \ldots, x_{\sigma(n)}\right)$ by $\mathbf{x}_{\sigma}$, and denote $\left(\alpha_{\sigma(1)}, \ldots, \alpha_{\sigma(n)}\right)$ by $\alpha_{\sigma}$. Then we give the following definition in the sense of Artin [1, 2].

Definition 1. Let $\alpha=\left(\alpha_{1}, \ldots, \alpha_{n}\right): I \longrightarrow F(X, \Gamma)$ be a path such that $\alpha(0)=\mathbf{x}$ and $\alpha(1)=\mathbf{x}_{\sigma}$ for some $\sigma \in \operatorname{Aut}(\Gamma)$. Then $c(\alpha)$ is called a crossing-changeable braid in $X \times I$. 
Remark 1. The crossing-changeable braids are quite different from ordinary braids in the sense of Artin. Indeed, there may be intersection points among all different strings in a crossingchangeable braid. In particular, some pairs of strings can intersect, and some pairs of strings cannot intersect strictly. This heavily depends upon the structure of $\Gamma$.

For example, let $\Gamma$ be the cycle graph $C_{4}$, then $\operatorname{Aut}(\Gamma)$ is the dihedral group $D_{8}=\langle a, b| a^{4}=$ $\left.b^{2}=e, b^{-1} a b=b^{-1}\right\rangle$, where a denotes the cyclic permutation (1234), and $b$ denotes the permutation (24).
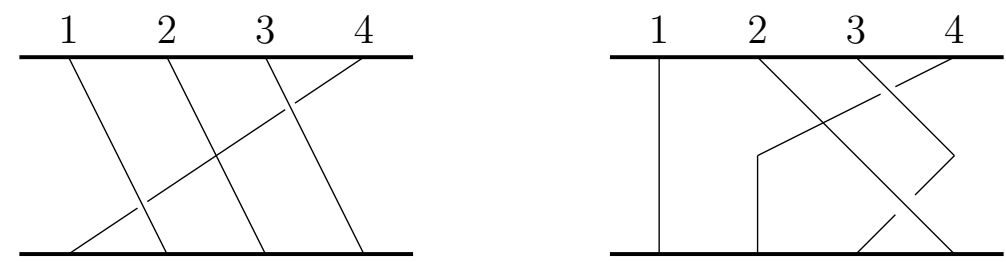

For two braids as shown in the figures above, we notice that the second and fourth strings can intersect since $\overline{24} \notin E(\Gamma)$. This is different from classical braids. However, we see that the first and fourth strings (the third and fourth strings respectively) can not intersect since $\overline{14}, \overline{34} \in E(\Gamma)$. This just agrees with classical braids.

By the construction of chromatic configuration spaces, obviously we can untie the intersection points appearing in the second and fourth strings of two braids as shown in the figures above by doing small deformations. Essentially nothing is changed up to homotopy.

The observation in Remark 1 gives us an insight to the equivalence of crossingchangeable braids. Set

$$
P(F(X, \Gamma), \mathbf{x}, \operatorname{Aut}(\Gamma)(\mathbf{x}))=\{\alpha: I \longrightarrow F(X, \Gamma) \mid \alpha(0)=\mathbf{x}, \text { and } \alpha(1) \in \operatorname{Aut}(\Gamma)(\mathbf{x})\}
$$

which consists of those paths with restricted endpoints in $F(X, \Gamma)$.

Definition 2. Let $\alpha$ and $\beta$ be two paths in $P(F(X, \Gamma), \mathbf{x}$, Aut $(\Gamma)(\mathbf{x}))$. We say that two corresponding crossing-changeable braids $c(\alpha)$ and $c(\beta)$ are equivalent, denoted by $c(\alpha) \sim c(\beta)$, if there exist $n$ homotopy maps $\widehat{h_{i}}: I \times I \longrightarrow X \times I$ given by $\widehat{h_{i}}(s, t)=\left(h_{i}(s, t), s\right), i=1, \ldots, n$, such that

(1) $\widehat{h_{i}}(s, 0)=c\left(\alpha_{i}\right)$ and $\widehat{h_{i}}(s, 1)=c\left(\beta_{i}\right)$;

(2) $h_{i}(0, t)=\alpha_{i}(0)=\beta_{i}(0)$ and $h_{i}(1, t)=\alpha_{i}(1)=\beta_{i}(1)$;

(3) For any $(s, t) \in I \times I$, if $\overline{j k} \in E(\Gamma)$ then $h_{j}(s, t) \neq h_{k}(s, t)$.

Actually, the equivalence of the crossing-changeable braids $c(\alpha)$ and $c(\beta)$ can be detected by the homotopy equivalence of the corresponding paths $\alpha$ and $\beta$.

Proposition 2.1. Let $\alpha$ and $\beta$ be two paths in $P(F(X, \Gamma), \mathbf{x}, \operatorname{Aut}(\Gamma)(\mathbf{x}))$. Then $\alpha \simeq \beta$ rel $\partial I$ if and only if $c(\alpha) \sim c(\beta)$.

Proof. Assume that $h=\left(h_{1}, \ldots, h_{n}\right): I \times I \longrightarrow F(X, \Gamma)$ is a homotopy relative to $\partial I$ from $\alpha$ to $\beta$. Then we can use $h$ to define $n$ homotopy maps

$$
\widehat{h_{i}}: I \times I \longrightarrow X \times I
$$

by $\widehat{h_{i}}(s, t)=\left(h_{i}(s, t), s\right)$, satisfying the three conditions of Definition 2 . Thus $c(\alpha)$ and $c(\beta)$ are equivalent. 
Conversely, suppose that $c(\alpha)$ and $c(\beta)$ are equivalent. Then there are $n$ homotopy maps

$$
\widehat{h_{i}}: I \times I \longrightarrow X \times I
$$

by $\widehat{h_{i}}(s, t)=\left(h_{i}(s, t), s\right)$, which satisfy the three conditions of Definition 2 . These $h_{i}{ }^{\prime} \mathrm{s}$ determine a map $h=\left(h_{1}, \ldots, h_{n}\right): I \times I \longrightarrow F(X, \Gamma)$, which is just the homotopy relative to $\partial I$ from $\alpha$ to $\beta$.

2.2. Crossing-changeable braid groups and extended fundamental groups. The concept of extended fundamental groups was given in a general way in [15, Section 4], and it plays an important role on the study of orbit braids.

By $\pi_{1}^{E}(F(X, \Gamma), \mathbf{x}, \operatorname{Aut}(\Gamma)(\mathbf{x}))$ we denote the extended fundamental group of $F(X, \Gamma)$ with action of group $\operatorname{Aut}(\Gamma)$ at the base point $\mathbf{x}$, which consists of the homotopy classes (relative to $\partial I$ ) of all paths in $P(F(X, \Gamma), \mathbf{x}, \operatorname{Aut}(\Gamma)(\mathbf{x}))$, with the operation $\bullet$ defined by

$$
[\alpha] \bullet[\beta]=\left[\alpha \circ \beta_{\sigma}\right]
$$

for two paths $\alpha$ and $\beta$ with $\alpha(1)=\mathbf{x}_{\sigma}$ and $\beta(1)=\mathbf{x}_{\tau}$ in $P(F(X, \Gamma), \mathbf{x}, \operatorname{Aut}(\Gamma)(\mathbf{x}))$, where $\circ$ is the usual operation between paths. Of course, generally $[\alpha] \bullet[\beta] \neq[\beta] \bullet[\alpha]$. Clearly, the fundament group $\pi_{1}(F(X, \Gamma), \mathbf{x})$ is a subgroup of $\pi_{1}^{E}(F(X, \Gamma), \mathbf{x}, \operatorname{Aut}(\Gamma)(\mathbf{x}))$. Note that all paths of $P(F(X, \Gamma), \mathbf{x}, \operatorname{Aut}(\Gamma)(\mathbf{x}))$ are not necessarily closed.

As mentioned before, all strings of each crossing-changeable braid $c(\alpha)$ at the base point $\mathbf{x}$ are unordered in $X \times I$, where $\alpha \in P(F(X, \Gamma)$, $\mathbf{x}$, Aut $(\Gamma)(\mathbf{x}))$. This means that for any $\sigma \in \operatorname{Aut}(\Gamma)$, actually $c(\alpha)=c\left(\alpha_{\sigma}\right)$ although generally $\alpha(0) \neq \alpha_{\sigma}(0)$ and $\alpha(1) \neq$ $\alpha_{\sigma}(1)$. We note that the endpoints $\alpha_{\sigma}(0)$ and $\alpha_{\sigma}(1)$ of $\alpha_{\sigma}$ are still in the orbit $\operatorname{Aut}(\Gamma)(\mathbf{x})$. Thus, the set

$$
\{c(\alpha) \mid \alpha \in P(F(X, \Gamma), \mathbf{x}, \operatorname{Aut}(\Gamma)(\mathbf{x}))\}
$$

denoted by $\mathcal{C}(X \times I, \operatorname{Aut}(\Gamma)(\mathbf{x}))$ consists of all possible crossing-changeable braids with endpoints lying in $\operatorname{Aut}(\Gamma)(\mathbf{x})$.

Geometrically, there is a natural operation on all crossing-changeable braids in $\mathcal{C}(X \times$ $I$, Aut $(\Gamma)(\mathbf{x}))$ by gluing the ending point of a crossing-changeable braid and the starting point of another crossing-changeable braid. This operation can also be defined in terms of the homotopy of paths. We state it as follows:

$$
c(\alpha) * c(\beta)=c\left(\alpha \circ \beta_{\sigma}\right)
$$

for two crossing-changeable braids $c(\alpha)$ and $c(\beta)$ with $\alpha(1)=\mathbf{x}_{\sigma}$ and $\beta(1)=\mathbf{x}_{\tau}$.

Let $B(X, \Gamma)$ denote the set formed by the equivalence classes of all crossing-changeable braids in $\mathcal{C}(X \times I, \operatorname{Aut}(\Gamma)(\mathbf{x}))$. Then the operation $*$ on $\mathcal{C}(X \times I, \operatorname{Aut}(\Gamma)(\mathbf{x}))$ induces the operation $\star$ on $B(X, \Gamma)$ as follows:

$$
[c(\alpha)] \star[c(\beta)]=[c(\alpha) * c(\beta)]=\left[c\left(\alpha \circ \beta_{\sigma}\right)\right]
$$

for two paths $\alpha$ and $\beta$ with $\alpha(1)=\mathbf{x}_{\sigma}$ and $\beta(1)=\mathbf{x}_{\tau}$ in $P(F(X, \Gamma), \mathbf{x}, \operatorname{Aut}(\Gamma)(\mathbf{x}))$.

It is not difficult to see that $B(X, \Gamma)$ forms a group under the operation $\star$, and generally it is not abelian.

Definition 3. The group $B(X, \Gamma)$ is called the crossing-changeable braid group of $F(X, \Gamma)$. Those classes $[c(\alpha)]$ with $\alpha(1)=\mathbf{x}$ in $B(X, \Gamma)$ form a subgroup, which is called the pure crossing-changeable braid group, denoted by $P(X, \Gamma)$. 
There is a homotopy description for these two braid groups.

\section{Theorem 2.1.}

(1) The crossing-changeable braid group $B(X, \Gamma)$ is isomorphic to the extended fundamental group $\pi_{1}^{E}(F(X, \Gamma), \mathbf{x}, \operatorname{Aut}(\Gamma)(\mathbf{x}))$;

(2) The pure crossing-changeable braid group $P(X, \Gamma)$ is isomorphic to the ordinary fundamental group $\pi_{1}(F(X, \Gamma), \mathbf{x})$.

Proof. Define the map $\varphi: \pi_{1}^{E}(F(X, \Gamma), \mathbf{x}, \operatorname{Aut}(\Gamma)(\mathbf{x})) \longrightarrow B(X, \Gamma)$ given by

$$
\varphi([\alpha])=[c(\alpha)] .
$$

For two paths $\alpha$ and $\beta$ with $\alpha(1)=\mathbf{x}_{\sigma}$ and $\beta(1)=\mathbf{x}_{\tau}$, a direct check shows that

$$
\varphi([\alpha] \bullet[\beta])=\varphi\left(\left[\alpha \circ \beta_{\sigma}\right]\right)=\left[c\left(\alpha \circ \beta_{\sigma}\right)\right][c(\alpha) * c(\beta)]=[c(\alpha)] \star[c(\beta)]=\varphi([\alpha]) \star \varphi([\beta])
$$

so $\varphi$ is a group homomorphism. Furthermore it follows from Proposition 2.1 that $\varphi$ is an isomorphism, as desired.

The isomorphism in (2) is just the restriction of $\varphi$ to $\pi_{1}(F(X, \Gamma), \mathbf{x})$.

Lemma 2.1. Each $[c(\alpha)]$ in $B(X, \Gamma)$ determines a unique element $\sigma \in \operatorname{Aut}(\Gamma)$, where $\alpha(1)=$ $\mathbf{x}_{\sigma}$.

Proof. This is a direct consequence of Proposition 2.1.

Theorem 2.2. There is the following natural exact sequence:

$$
1 \longrightarrow P(X, \Gamma) \longrightarrow B(X, \Gamma) \longrightarrow \operatorname{Aut}(\Gamma) \longrightarrow 1
$$

Proof. Take a class $[c(\alpha)]$ in $B(X, \Gamma)$, by Lemma 2.1, there is a unique element $\sigma \in$ $\operatorname{Aut}(\Gamma)$ such that $\alpha(1)=\mathbf{x}_{\sigma}$. Define the map $\Phi: B(X, \Gamma) \longrightarrow \operatorname{Aut}(\Gamma)$ given by

$$
\Phi([c(\alpha)])=\sigma .
$$

For two paths $\alpha$ and $\beta$ with $\alpha(1)=\mathbf{x}_{\sigma}$ and $\beta(1)=\mathbf{x}_{\tau}$ in $P(F(X, \Gamma), \mathbf{x}, \operatorname{Aut}(\Gamma)(\mathbf{x}))$, it is easy to see that $\alpha \circ \beta_{\sigma}(1)=\mathbf{x}_{\sigma \tau}$, so $\Phi([c(\alpha)] \star[c(\beta)])=\Phi\left(\left[c\left(\alpha \circ \beta_{\sigma}\right)\right]=\sigma \tau\right.$. Thus $\Phi$ is a group homomorphism.

Choose an element $\sigma$ in $\operatorname{Aut}(\Gamma)$. Since $F(X, \Gamma)$ is connected, there must be a path $\alpha: I \longrightarrow F(X, \Gamma)$ such that $\alpha(0)=\mathbf{x}$ and $\alpha(1)=\mathbf{x}_{\sigma}$, so $[c(\alpha)] \in B(X, \Gamma)$. This means that $\Phi$ is surjective.

For $[c(\alpha)] \in B(X, \Gamma)$, if $\Phi([c(\alpha)])$ is the identity of $\operatorname{Aut}(\Gamma)$, then $\alpha(1)=\mathbf{x}$, so $\operatorname{ker}(\Phi)$ is isomorphic to $P(X, \Gamma)$. This completes the proof.

2.3. Associated with classical configuration space $F(X, n)$. As a subspace of $F(X, \Gamma)$, the classical configuration space $F(X, n)$ admits a free action of the symmetric group $\Sigma_{n}$. Thus, for any non-trivial subgroup $G$ of $\Sigma_{n}, G$ can still act on $F(X, n)$ freely. By [15, Section 4] we may define the extended fundamental group at the base point $\mathbf{x}$ :

$$
\pi_{1}^{E}(F(X, n), \mathbf{x}, G(\mathbf{x}))=\{[\alpha] \mid \alpha: I \longrightarrow F(X, n) \text { with } \alpha(0)=\mathbf{x} \text { and } \alpha(1) \in G(\mathbf{x})\}
$$

with the operation given by

$$
[\alpha] \bullet[\beta] \underset{7}{=}\left[\alpha \circ \beta_{\sigma}\right]
$$


where $\sigma \in G \subset \Sigma_{n}$ is the unique element determined by $\alpha$ such that $\alpha(1)=\mathbf{x}_{\sigma}$. Then we know from [15] that there is the following short exact sequence

$$
1 \longrightarrow \pi_{1}(F(X, n), \mathbf{x}) \longrightarrow \pi_{1}^{E}(F(X, n), \mathbf{x}, G(\mathbf{x})) \longrightarrow G \longrightarrow 1 .
$$

Since $G$ is finite and the action of $G$ on $F(X, n)$ is free, by [15, Section 4, (B)] we have that the group $\pi_{1}^{E}(F(X, n), \mathbf{x}, G(\mathbf{x}))$ is isomorphic to the fundamental group $\pi_{1}(F(X, n) / G, \overline{\mathbf{x}})$, where $\overline{\mathrm{x}}$ is the image of $\mathrm{x}$ under the projection $F(X, n) \longrightarrow F(X, n) / G$.

On the other hand, since $G(\mathbf{x}) \subset \Sigma_{n}(\mathbf{x})$, it is easy to see that $\pi_{1}^{E}(F(X, n), \mathbf{x}, G(\mathbf{x})) \cong$ $\pi_{1}(F(X, n) / G, \overline{\mathbf{x}})$ is a subgroup of $\pi_{1}^{E}\left(F(X, n), \mathbf{x}, \Sigma_{n}(\mathbf{x})\right) \cong \pi_{1}\left(F(X, n) / \Sigma_{n}, \widetilde{\mathbf{x}}\right)$. It is wellknown that $\pi_{1}\left(F(X, n) / \Sigma_{n}, \widetilde{\mathbf{x}}\right)$ is regarded as the braid group $B(X, n)$ in $X \times I$. Therefore, $\pi_{1}^{E}(F(X, n), \mathbf{x}, G(\mathbf{x})) \cong \pi_{1}(F(X, n) / G, \overline{\mathbf{x}})$ is a subgroup of $B(X, n)$, also denoted by $\left.B(X, n)\right|_{G}$. Of course, this can also be seen from the theory of covering spaces.

Now, choose $G$ as the subgroup $\operatorname{Aut}(\Gamma)$ of $\Sigma_{n}$, let us discuss the relation between $B(X, \Gamma)$ and $\left.B(X, n)\right|_{\text {Aut }(\Gamma)}$. First, consider the natural inclusion

$$
i: F(X, n) \hookrightarrow F(X, \Gamma) .
$$

Lemma 2.2. The induced map $i_{*}: \pi_{1}(F(X, n), \mathbf{x}) \rightarrow \pi_{1}(F(X, \Gamma), \mathbf{x})$ is an epimorphism.

Proof. Take a path class $[\alpha] \in \pi_{1}(F(X, \Gamma), \mathbf{x})$. For any $s \in I$, if $\alpha(s) \in F(X, n)$, then $[\alpha]$ is also an element of $\pi_{1}(F(X, n), \mathbf{x})$. Otherwise, there may be some $\alpha_{j}$ and $\alpha_{k}$ as paths from $I$ to $X$ with intersection points, where $\alpha_{j}$ and $\alpha_{k}$ are two coordinates in $\alpha=\left(\alpha_{1}, \ldots, \alpha_{n}\right)$. Using the same idea as in the proof of [15, Lemma 2.7], we can do a slight homotopy deformation on $\alpha$ without touching endpoints to produce a new path $\alpha^{\prime}$ such that all coordinates of $\alpha^{\prime}$ do not intersect each other, implying that $\left[\alpha^{\prime}\right] \in \pi_{1}(F(X, n), \mathbf{x})$. This deformation makes sure that $\alpha \simeq \alpha^{\prime}$ rel $\partial I$ in $F(X, \Gamma)$. Thus $i_{*}\left(\left[\alpha^{\prime}\right]\right)=\left[\alpha^{\prime}\right]=[\alpha]$.

Remark 2. In the proof of Lemma 2.2, when doing various different slight homotopy deformations on the path $\alpha$ in $F(X, \Gamma)$, we can obtain many new paths. Generally these new paths may not be the same up to homotopy in $F(X, n)$, but they are always the same up to homotopy in $F(X, \Gamma)$.

Since $\operatorname{Aut}(\Gamma)$ can freely act on $F(X, n)$, it is not difficult to see that the inclusion $i: F(X, n) \hookrightarrow F(X, \Gamma)$ becomes an Aut $(\Gamma)$-map, and it also induces the homomorphism

$$
i_{*}: \pi_{1}^{E}(F(X, n), \mathbf{x}, \operatorname{Aut}(\Gamma)(\mathbf{x})) \rightarrow \pi_{1}^{E}(F(X, \Gamma), \mathbf{x}, \operatorname{Aut}(\Gamma)(\mathbf{x})) .
$$

The restriction map of this homomorphism to $\pi_{1}(F(X, n), \mathbf{x})$ is just the epimorphism in Lemma 2.2. Actually this homomorphism without any restriction is still an epimorphism.

Lemma 2.3. The homomorphism $i_{*}: \pi_{1}^{E}(F(X, n), \mathbf{x}, \operatorname{Aut}(\Gamma)(\mathbf{x})) \rightarrow \pi_{1}^{E}(F(X, \Gamma), \mathbf{x}, \operatorname{Aut}(\Gamma)(\mathbf{x}))$ is surjective.

Proof. The proof is a similar argument to one of Lemma 2.2 .

Together with Theorems 2.1-2.2 and Lemmas 2.2-2.3, we have 
Proposition 2.2. There is an epimorphism between two short exact sequences.

$1 \longrightarrow \pi_{1}(F(X, n), \mathbf{x}) \longrightarrow \pi_{1}^{E}(F(X, n), \mathbf{x}, \operatorname{Aut}(\Gamma)(\mathbf{x})) \longrightarrow \operatorname{Aut}(\Gamma) \longrightarrow 1$

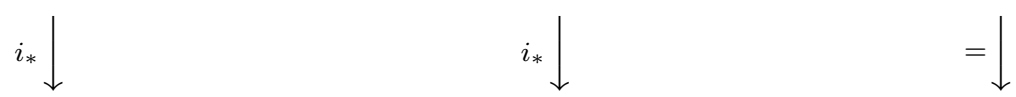

$1 \longrightarrow \pi_{1}(F(X, \Gamma), \mathbf{x}) \longrightarrow \pi_{1}^{E}(F(X, \Gamma), \mathbf{x}, \operatorname{Aut}(\Gamma)(\mathbf{x})) \longrightarrow \operatorname{Aut}(\Gamma) \longrightarrow 1$

or in terms of braid groups

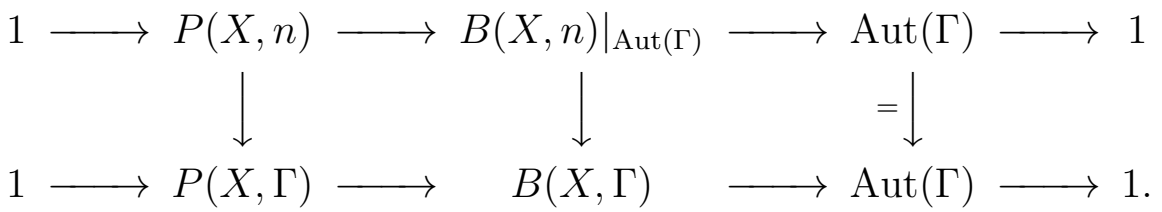

3. Calculations of $B(\mathbb{C}, \Gamma)$ And $P(\mathbb{C}, \Gamma)$

In this section we will focus on the case $X=\mathbb{C}$. We will discuss the structure of $B(\mathbb{C}, \Gamma)$ and $P(\mathbb{C}, \Gamma)$, we abbreviate them to $B(\Gamma)$ and $P(\Gamma)$. When $\Gamma$ is the complete graph $K_{n}$ on vertex set $[n], B(\Gamma)$ and $P(\Gamma)$ just are the classical braid group $B_{n}$ and pure braid group $P_{n}$, respectively. It is well-known that Artin [2] and Markoff [17] gave the presentations of $B_{n}$ and $P_{n}$ respectively, which are stated as follows.

Theorem 3.1 (Artin). The braid group $B_{n}$ is generated by $\sigma_{i}, i=1, \ldots, n-1$ with the relations

$$
\left\{\begin{array}{l}
\sigma_{i} \sigma_{j}=\sigma_{j} \sigma_{i} \text { for }|i-j|>1 \\
\sigma_{i} \sigma_{i+1} \sigma_{i}=\sigma_{i+1} \sigma_{i} \sigma_{i+1}
\end{array}\right.
$$

where $\sigma_{i}$ is the braid with only one crossing, as shown below

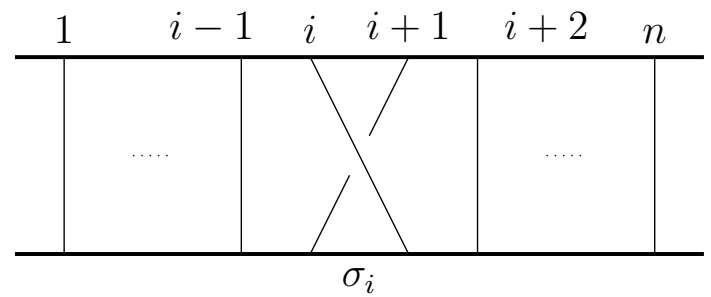

Let $s_{i, j}, 1 \leq i<j \leq n$ be the elements of $B_{n}$ given by the formulae

$$
s_{i, j}=\sigma_{j-1} \ldots \sigma_{i+1} \sigma_{i}^{2} \sigma_{i+1}^{-1} \ldots \sigma_{j-1}^{-1}
$$

as shown below

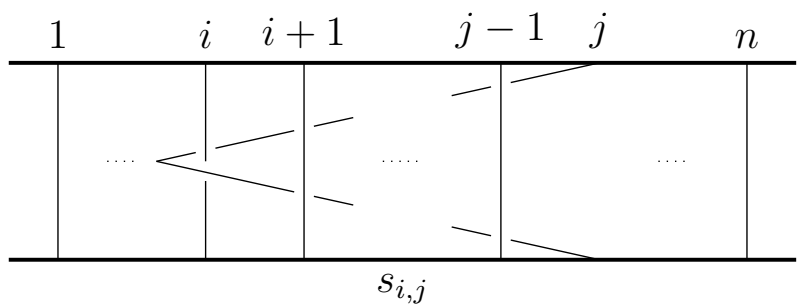

Figure A

Theorem 3.2 (Markoff). The elements $s_{i, j}, 1 \leq i<j \leq n$ with the Burau relations:

(1) $s_{i, j} s_{k, l}=s_{k, l} s_{i, j}$

(2) $s_{i, j} s_{i, k} s_{j, k}=s_{i, k} s_{j, k} s_{i, j}=s_{j, k} s_{i, j} s_{i, k}$

for $i<j<k<l$ and for $i<k<l<j$, for $i<j<k$, 
(3) $s_{i, k} s_{j, k} s_{j, l} s_{j, k}^{-1}=s_{j, k} s_{j, l} s_{j, k}^{-1} s_{i, k} \quad$ for $i<j<k<l$.

give a presentation of pure braid group $P_{n}$.

Remark 3. Intuitively we see that each generator in $B_{n}$ and $P_{n}$ corresponds to an edge in $K_{n}$. In particular, all generators of $P_{n}$ bijectively correspond to all edges of $K_{n}$.

3.1. The presentation of $P(\Gamma)$. Let $\Gamma$ be a simple graph without loops on vertex set $[n]$. Now let us discuss the the presentation of $P(\Gamma)$, which is the generalization of $P_{n}$.

Define $s_{i j}$ to be the braid in $P(\Gamma)$ with the $i$-th string and the $j$-th string tangled one time, as shown in Figure A. Obviously, if $\overline{i j} \notin E(\Gamma)$, then $s_{i j}$ will be trivial. Let $\Delta_{i j k}$ denote the 3-circuit in $K_{n}$, which is formed by three edges $\overline{i j}, \overline{i k}, \overline{j k}$ where $i, j, k \in[n]$. Let $\square_{i j k l}$ denote the 4-circuit in $K_{n}$, which is formed by four edges $\overline{i j}, \overline{j k}, \overline{k l}, \overline{i l}$ where $i, j, k, l \in[n]$.

Theorem 3.3. The elements $s_{i, j}, \overline{i j} \in E(\Gamma)$ with the following relations

(1) if $\overline{i j}, \overline{k l} \in E\left(\square_{i j k l}\right) \cap E(\Gamma)$ with $i<j<k<l$ or $i<k<l<j$, then

$$
s_{i, j} s_{k, l}=s_{k, l} s_{i, j}
$$

$\left(2_{1}\right)$ if $\Delta_{i j k}$ with $i<j<k$ is a 3-circuit in $\Gamma$, then

$$
s_{i, j} s_{i, k} s_{j, k}=s_{i, k} s_{j, k} s_{i, j}=s_{j, k} s_{i, j} s_{i, k}
$$

$\left(2_{2}\right)$ if $\Delta_{i j k}$ is not a 3-circuit in $\Gamma$ but $\overline{i j}, \overline{j k} \in E(\Gamma)$ (so $\overline{i k} \notin E(\Gamma)$ ), then

$$
s_{i, j} s_{j, k}=s_{j, k} s_{i, j}
$$

where the restriction condition $i<j<k$ is not necessarily satisfied;

$\left(3_{1}\right)$ if $\overline{i k}, \overline{j l} \in E\left(\square_{i j k l}\right) \cap E(\Gamma)$ with $i<j<k<l$ and $\Delta_{j k l}$ is a 3-circuit in $\Gamma$, then

$$
s_{i, k} s_{j, k} s_{j, l} s_{j, k}^{-1}=s_{j, k} s_{j, l} s_{j, k}^{-1} s_{i, k}
$$

$\left(3_{2}\right)$ if $\overline{i k}, \overline{j l} \in E\left(\square_{i j k l}\right) \cap E(\Gamma)$ with $i<j<k<l$ but $\Delta_{j k l}$ is not a 3-circuit in $\Gamma$, then

$$
s_{i, k} s_{j, l}=s_{j, l} s_{i, k}
$$

give a presentation of the pure crossing-changable braid group $P(\Gamma)$.

Proof. We know from Proposition 2.2 that the induced map $i_{*}: P_{n} \longrightarrow P(\Gamma)$ by the inclusion $i: F(\mathbb{C}, n) \hookrightarrow F(\mathbb{C}, \Gamma)$ is an epimorphism. This means that all elements $s_{i, j}$, $\overline{i j} \in E(\Gamma)$, form a system of generators in $P(\Gamma)$, and all possible relations among them can be obtained by the images of the epimorphism $i_{*}$ acting on the Burau relations (1)-(3) in Theorem 3.2. Therefore, the required relations follow easily from direct calculations.

Remark 4. Randell tells us in [19, 20] that there is also another approach to get Theorem 3.3 from the viewpoint of complex hyperplane arrangements. Actually $F(\mathbb{C}, \Gamma)$ can be regarded as a complement space of complex hyperplane arrangements as follows: Let $H_{i, j}:=\left\{\left(z_{1}, \ldots, z_{n}\right) \in\right.$ $\left.\mathbb{R}^{2 n} \mid z_{i}=z_{j}\right\}$. Then $F(\mathbb{C}, \Gamma)=\mathbb{C}^{n} \backslash \bigcup_{\overline{i j} \in E(\Gamma)} H_{i, j}$. Randell's method is more geometric, for more details, see [19, 20]). Here the method used is of the combinatorial nature.

Corollary 1. If $\Gamma$ doesn't contain any 3-circuit, then $P(\Gamma)$ is a free abelian group generated by $s_{i, j}, \overline{i j} \in E(\Gamma)$. 
3.2. The presentation of $\mathbf{B}(\Gamma)$. The group structure of $B(\Gamma)$ heavily relies on $\operatorname{Aut}(\Gamma)$. However, as pointed out in [5], the group structure of $\operatorname{Aut}(\Gamma)$ is still an unsolved problem except for some particular classes of graphs. Furthermore, we see from the short exact sequence

$$
1 \rightarrow P(\Gamma) \rightarrow B(\Gamma) \rightarrow \operatorname{Aut}(\Gamma) \rightarrow 1
$$

(see Theorem 2.2) that the determination of $B(\Gamma)$ would be quite difficult in general although a presentation of $P(\Gamma)$ has been given in Theorem 3.3 .

For a short exact sequence of groups

$$
1 \rightarrow A \stackrel{j}{\rightarrow} E \stackrel{p}{\rightarrow} G \rightarrow 1
$$

the group extension theory [18] tells us how to give a presentation of $E$ from the given $A=\langle S \mid R\rangle$ and $G=\langle T \mid Q\rangle$. Choose a mapping (not necessarily a homomorphism) $\psi$ : $G \rightarrow E$ with $p \circ \psi=\mathrm{id}_{G}$. Clearly the choice of $\psi$ is not unique since $p$ is surjective. Then we use $\psi$ to give a conjugation action $\gamma$ of $G$ on $A$ defined by $\gamma(g, a)=\psi(g)^{-1} a \psi(g)$ where $g \in G$ and $a \in A$. For each $t \in T, \gamma(t, \cdot)$ defines an automorphism $\gamma_{t}: A \rightarrow A$. Moreover, $E$ is presented as follows

$$
\left\langle S \cup \psi(T) \mid R \cup\left\{\psi(t)^{-1} s \psi(t)=\gamma_{t}(s): s \in S, t \in T\right\} \cup\left\{\omega_{q}(s)=q(\psi(t) ; t \in T): q \in Q\right\}\right\rangle
$$

where $w_{q}(s)$ is a word in $S$ such that $j\left(\omega_{q}(s)\right)=q(\psi(t))$ in $E$, and each $q \in Q$ corresponds to a word $q(t)$ equal to the identity $e_{G}$ in $G$ so $p\left(q(\psi(t))=e_{G}\right.$.

3.2.1. A presentation of $B\left(C_{n}\right)$. With the above understanding, now we consider the case in which $\Gamma$ is the cycle graph $C_{n}$ in $K_{n}$. Without loss of generality, assume that $C_{n}$ is the cycle graph formed by edges $\overline{12}, \overline{23}, \ldots, \overline{n(n-1)}$ and $\overline{1 n}$. Then $\operatorname{Aut}\left(C_{n}\right)$ is the dihedral group

$$
\mathcal{D}_{2 n}=\left\langle a, b \mid a^{n}=b^{2}=e, b a b=a^{-1}\right\rangle
$$

where $a$ denotes the cyclic permutation $(12 \cdots n)$ and $b$ denotes the permutation

$$
b=(2 n)(3 n-1) \cdots(r(n) s(n))
$$

where $r(n)=\left\{\begin{array}{ll}\frac{n}{2} & \text { if } n \text { is even } \\ \frac{n+1}{2} & \text { if } n \text { is odd }\end{array}\right.$ and $s(n)= \begin{cases}\frac{n+4}{2} & \text { if } n \text { is even } \\ \frac{n+3}{2} & \text { if } n \text { is odd }\end{cases}$

By Corollary 1, $P\left(C_{n}\right)$ is a free abelian group generated by $s_{i, i+1}(1 \leq i \leq n-1)$ and $s_{1, n}$, so

$$
P\left(C_{n}\right) \cong \bigoplus_{1 \leq i \leq n-1} \mathbb{Z}\left(s_{i, i+1}\right) \oplus \mathbb{Z}\left(s_{1, n}\right)
$$

Next we are going to give a presentation of $B\left(C_{n}\right)$ by making use of the group extension theory from the short exact sequence

$$
1 \rightarrow P\left(C_{n}\right) \stackrel{j}{\rightarrow} B\left(C_{n}\right) \stackrel{p}{\rightarrow} \mathcal{D}_{2 n} \rightarrow 1
$$


By Proposition 2.2, we have the following commutative diagram with three epimorphisms

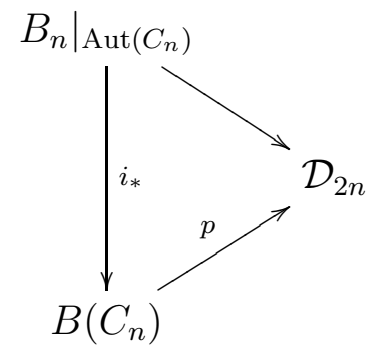

Choose a map $\psi: \mathcal{D}_{2 n} \longrightarrow B\left(C_{n}\right)$ with $p \circ \psi=\operatorname{id}_{\mathcal{D}_{2 n}}$, defined by $\psi(a)=i_{*}\left(a_{1, n}\right)$ and

$$
\psi(b)=i_{*}\left(a_{2, n} a_{3, n}^{-1} a_{3, n-1} a_{4, n-1}^{-1} \cdots a_{r(n), s(n)} a_{r(n)+1, s(n)}^{-1}\right)
$$

where $a_{i, j}$ denotes the word $\sigma_{j-1} \cdots \sigma_{i}$ in $B_{n}$ (for the meanings of $\sigma_{i}{ }^{\prime}$ s, see Theorem 3.1); namely

$$
a_{i, j}=\sigma_{j-1} \cdots \sigma_{i} .
$$

Geometrically, $a_{k, l} a_{k+1, l}^{-1}$ expresses the braid of exchanging the $k$-th and $l$-th strings, which is abbreviated as $e_{k, l}$ for convenience. Then

$$
\psi(b)=i_{*}\left(e_{2, n} e_{3, n-1} \cdots e_{r(n), s(n)}\right) .
$$

Remark 5. Since $i_{*}:\left.B_{n}\right|_{\operatorname{Aut}\left(C_{n}\right)} \longrightarrow B\left(C_{n}\right)$ is induced by the conclusion $i: F(\mathbb{C}, n) \hookrightarrow$ $F\left(\mathbb{C}, C_{n}\right)$, it is easy to see that for any braid $\rho$ in $\left.B_{n}\right|_{\operatorname{Aut}\left(C_{n}\right)}, i_{*}(\rho)$ can be understood as the braid of $\rho$ in $B\left(C_{n}\right)$, so we may write $i_{*}(\rho)=\rho$.

First let us prove some useful equations in $B_{n}$.

Lemma 3.1. In $B_{n}$, the following equations hold:

$\left(1^{\circ}\right) \sigma_{i+1} \sigma_{i} \sigma_{i+1}^{2}=\sigma_{i}^{2} \sigma_{i+1} \sigma_{i}$;

$\left(2^{\circ}\right) a_{i, j} \sigma_{k}=\sigma_{k-1} a_{i, j}$ for $i<k<j$

$\left(3^{\circ}\right) a_{i, j} a_{k, l}=a_{k-1, l-1} a_{i, j}$ for $i<k<l \leq j$;

$\left(4^{\circ}\right) e_{2, n} e_{3, n-1}, \ldots, e_{r(n), s(n)}$ are pairwise commutative;

$\left(5^{\circ}\right) e_{k, n+2-k} a_{1, n}=a_{1, n} e_{k+1, n+3-k}$ for $2<k \leq\left[\frac{n+1}{2}\right]$.

Proof. Making use of the relations (1)-(2) in Theorem 3.1, direct calculations give

$$
\sigma_{i+1} \sigma_{i} \sigma_{i+1}^{2}=\sigma_{i+1} \sigma_{i} \sigma_{i+1} \sigma_{i+1}=\sigma_{i} \sigma_{i+1} \sigma_{i} \sigma_{i+1}=\sigma_{i} \sigma_{i} \sigma_{i+1} \sigma_{i}=\sigma_{i}^{2} \sigma_{i+1} \sigma_{i}
$$

and

$$
\begin{aligned}
a_{i, j} \sigma_{k} & =\sigma_{j-1} \ldots \sigma_{k} \sigma_{k-1} \ldots \sigma_{i} \sigma_{k}=\sigma_{j-1} \ldots \sigma_{k} \sigma_{k-1} \sigma_{k} \ldots \sigma_{i} \\
& =\sigma_{j-1} \ldots \sigma_{k-1} \sigma_{k} \sigma_{k-1} \ldots \sigma_{i}=\sigma_{k-1} \sigma_{j-1} \ldots \sigma_{i} \\
& =\sigma_{k-1} a_{i, j} .
\end{aligned}
$$

This proves $\left(1^{\circ}\right)$ and $\left(2^{\circ}\right)$. Using the equation $\left(2^{\circ}\right)$, we have

$$
a_{i, j} a_{k, l}=a_{i, j} \sigma_{l-1} \cdots \sigma_{k}=\sigma_{l-2} \cdots \sigma_{k-1} a_{i, j}=a_{k-1, l-1} a_{i, j}
$$


as desired in $\left(3^{\circ}\right)$. On $\left(4^{\circ}\right)$, for arbitrary $k<l$, repeating the use of the equation $\left(3^{\circ}\right)$, we have

$$
\begin{aligned}
e_{k, n+2-k} e_{l, n+2-l} & =a_{k, n+2-k} a_{k+1, n+2-k}^{-1} a_{l, n+2-l} a_{l+1, n+2-l}^{-1} \\
& =a_{k, n+2-k} a_{l+1, n+3-l} a_{k+1, n+2-k}^{-1} a_{l+1, n+2-l}^{-1} \\
& =a_{l, n+2-l} a_{k, n+2-k} a_{l+2, n+3-l}^{-1} a_{k+1, n+2-k}^{-1} \\
& =a_{l, n+2-l} a_{l+1, n+2-l}^{-1} a_{k, n+2-k} a_{k+1, n+2-k}^{-1} \\
& =e_{l, n+2-l} e_{k, n+2-k}
\end{aligned}
$$

as desired. On $\left(5^{\circ}\right)$, when $2<k \leq\left[\frac{n+1}{2}\right]$, repeating the use of the equation $\left(3^{\circ}\right)$ gives

$$
\begin{aligned}
e_{k, n+2-k} a_{1, n} & =a_{k, n+2-k} a_{k+1, n+2-k}^{-1} a_{1, n} \\
& =a_{k, n+2-k} a_{1, n} a_{k+2, n+3-k}^{-1}=a_{1, n} a_{k+1, n+3-k} a_{k+2, n+3-k}^{-1} \\
& =a_{1, n} e_{k+1, n+3-k} .
\end{aligned}
$$

Proposition 3.1. The elements $s_{i, j}, \overline{i j} \in E\left(C_{n}\right), \psi(a)$ and $\psi(b)$ with three families of relations: $R_{1}: s_{i, j}, \overline{i j} \in E\left(C_{n}\right)$ are pairwise commutative;

$$
\begin{gathered}
R_{2}: \psi(a)^{-1} s_{i, j} \psi(a)= \begin{cases}s_{a(i), a(j)} & \text { if } 1 \leq i<n-1 \text { and } j=i+1 \\
s_{a(j), a(i)} & \text { if } i=1 \text { and } j=n \\
s_{a(j), a(i)} & \text { if } i=n-1 \text { and } j=n ;\end{cases} \\
\psi(b)^{-1} s_{i, j} \psi(b)= \begin{cases}s_{b(j), b(i)} & \text { if } 1<i \leq n-1 \text { and } j=i+1 \\
s_{b(i), b(j)} & \text { if } i=1 \text { and } j=2 \\
s_{b(i), b(j)} & \text { if } i=1 \text { and } j=n ;\end{cases} \\
R_{3}: \psi(a)^{n}=s_{1, n} s_{n-1, n} s_{n-2, n-1} \cdots s_{1,2} ;
\end{gathered}
$$

give a presentation of the crossing-changable braid group $B\left(C_{n}\right)$.

Proof. By the theory of group extension, it suffices to show that three families of relations $R_{1}-R_{3}$ hold.

The relation $R_{1}$ directly follows from the commutativity of $P\left(C_{n}\right)$.

The relation $R_{2}$ comes from the conjugation action of $\mathcal{D}_{2 n}$ on $P\left(C_{n}\right)$. We convention that $\overline{i j} \in E\left(C_{n}\right)$ means for $1 \leq i \leq n-1, j=i+1$ and for $i=n, j=1$. We proceed as 
follows. On $\psi(a)^{-1} s_{i, j} \psi(a)$ with $\overline{i j} \in E\left(C_{n}\right)$,

$$
\begin{aligned}
\psi(a)^{-1} s_{i, j} \psi(a) & =\psi(a)^{-1} \sigma_{i}^{2} \psi(a)=i_{*}\left(a_{1, n}^{-1} \sigma_{i}^{2} a_{1, n}\right) \\
& =\left\{\begin{array}{ll}
i_{*}\left(a_{1, n}^{-1} a_{1, n} \sigma_{i+1}^{2}\right) & \text { for } 1 \leq i \leq n-2 \\
i_{*}\left(a_{1, n} \sigma_{1}^{2} a_{1, n}^{-1}\right) & \text { for } i=n-1 \\
i_{*}\left(a_{1, n}^{-1} a_{2, n} \sigma_{1}^{2} a_{2, n}^{-1} a_{1, n}\right) & \text { for } i=n
\end{array} \quad \text { (by Lemma 3.1 }\left(1^{\circ}\right)-\left(2^{\circ}\right)\right) \\
& =\left\{\begin{array}{ll}
s_{i+1, i+2} & \text { for } 1 \leq i \leq n-2 \\
s_{1, n} & \text { for } i=n-1 \\
s_{1,2} & \text { for } i=n
\end{array} \text { (by (‥1) } \text { and (3.2) }\right) \\
= & s_{a(i), a(j) .}
\end{aligned}
$$

On $\psi(b)^{-1} s_{i, j} \psi(b)$ with $\overline{i j} \in E\left(C_{n}\right)$, we have that

$$
\psi(b)^{-1} s_{i, j} \psi(b)= \begin{cases}e_{\frac{n}{2}, \frac{n+4}{2}}^{-1} \cdots e_{2, n}^{-1} s_{i, j} e_{2, n} \cdots e_{\frac{n}{2}, \frac{n+4}{2}} & \text { if } n \text { is even } \\ e_{\frac{n+1}{2}, \frac{n+3}{2}}^{-1} \cdots e_{2, n}^{-1} s_{i, j} e_{2, n} \cdots e_{\frac{n+1}{2}, \frac{n+3}{2}} & \text { if } n \text { is odd }\end{cases}
$$

In particular, if $\overline{i j} \notin E\left(C_{n}\right)$ then $s_{i, j}$ is a trivial braid so $\psi(b)^{-1} s_{i, j} \psi(b)=e$. First let us look at the conjugation $e_{k, n+2-k}^{-1} s_{i, j} e_{k, n+2-k}$ where $1<k \leq\left[\frac{n+1}{2}\right]$ and $\overline{i j} \in E\left(C_{n}\right)$. If each of $i$ and $j$ is not equal to $k$ or $n+2-k$, then we easily see from the geometric meanings of $e_{k, n+2-k}$ and $s_{i, j}$ that $e_{k, n+2-k}$ and $s_{i, j}$ are commutative so

$$
e_{k, n+2-k}^{-1} s_{i, j} e_{k, n+2-k}=s_{i, j}
$$

Thus, by Lemma 3.1 $\left(4^{\circ}\right)$, it suffices to consider the case in which one of $i$ and $j$ is equal to $k$ or $n+2-k$. The argument proceeds as follows:

When $i=1, j=2$,

$$
\begin{aligned}
\psi(b)^{-1} s_{1,2} \psi(b) & =i_{*}\left(e_{2, n}^{-1} s_{1,2} e_{2, n}\right)=i_{*}\left(a_{3, n} a_{2, n}^{-1} \sigma_{1}^{2} a_{2, n} a_{3, n}^{-1}\right)=i_{*}\left(a_{3, n} \sigma_{2}^{-1} \sigma_{1}^{2} \sigma_{2} a_{3, n}^{-1}\right) \\
& =i_{*}\left(s_{2, n}^{-1} s_{1, n} s_{2, n}\right)=s_{1, n}=s_{b(1), b(2)} .
\end{aligned}
$$

When $i=1, j=n$,

$\psi(b)^{-1} s_{1, n} \psi(b)=i_{*}\left(e_{2, n}^{-1} s_{1, n} e_{2, n}\right)=i_{*}\left(a_{3, n} a_{2, n}^{-1} a_{2, n} \sigma_{1}^{2} a_{2, n}^{-1} a_{2, n} a_{3, n}^{-1}\right)=i_{*}\left(\sigma_{1}^{2}\right)=s_{1,2}=s_{b(1), b(n)}$. 
When $i=l-1, j=l$ where $2<l \leq n$, if $2<l \leq\left[\frac{n+1}{2}\right]$ then

$$
\begin{aligned}
\psi(b)^{-1} s_{l-1, l} \psi(b)= & i_{*}\left(e_{l-1, n+3-l}^{-1} e_{l, n+2-l}^{-1} s_{l-1, l} e_{l, n+2-l} e_{l-1, n+3-l}\right) \\
= & i_{*}\left(e_{l-1, n+3-l}^{-1} a_{l+1, n+2-l} a_{l, n+2-l}^{-1} s_{l-1, l} a_{l, n+2-l} a_{l+1, n+2-l}^{-1} e_{l-1, n+3-l}\right) \\
= & i_{*}\left(e_{l-1, n+3-l}^{-1} a_{l+1, n+2-l} \sigma_{l}^{-1} s_{l-1, l} \sigma_{l} a_{l+1, n+2-l}^{-1} e_{l-1, n+3-l}\right) \\
= & i_{*}\left(e_{l-1, n+3-l}^{-1} s_{l, n+2-l}^{-1} s_{l-1, n+2-l} s_{l, n+2-l} e_{l-1, n+3-l}\right) \\
= & i_{*}\left(s_{l, n+2-l}^{-1} e_{l-1, n+3-l}^{-1} s_{l-1, n+2-l} e_{l-1, n+3-l} s_{l, n+2-l}\right) \\
= & i_{*}\left(s_{l, n+2-l}^{-1} a_{l-1, n+3-l}^{-1} a_{l-1, n+2-l} a_{l-1, n+1-l}^{-1} \sigma_{n+1-l}^{2} a_{l-1, n+1-l} a_{l-1, n+2-l}^{-1}\right. \\
& \left.\cdot a_{l-1, n+3-l} s_{l, n+2-l}\right) \\
= & i_{*}\left(s_{l, n+2-l}^{-1} a_{l-1, n+3-l}^{-1} \sigma_{n+1-l}^{2} a_{l-1, n+3-l} s_{l, n+2-l}\right) \\
= & i_{*}\left(s_{l, n+2-l}^{-1} a_{l-1, n+1-l}^{-1} \sigma_{n+1-l}^{-1} \sigma_{n+1-l} \sigma_{n+2-l}^{2} \sigma_{n+1-l}^{-1} \sigma_{n+1-l} a_{l-1, n+1-l} s_{l, n+2-l}\right) \\
= & i_{*}\left(s_{l, n+2-l}^{-1} s_{n+2-l, n+3-l} s_{l, n+2-l}\right)=s_{n+2-l, n+3-l}=s_{b(l-1), b(l)} ;
\end{aligned}
$$

if $\left[\frac{n+1}{2}\right]<l \leq n$, in the similar way as above, we can still obtain $\psi(b)^{-1} s_{l-1, l} \psi(b)=$ $s_{b(l-1), b(l)}$.

In conclusion, $\psi(b)^{-1} s_{i, j} \psi(b)=\left\{\begin{array}{ll}s_{b(j), b(i)} & \text { if } 1<i \leq n-1 \text { and } j=i+1 \\ s_{b(i), b(j)} & \text { if } i=1 \text { and } j=2 \\ s_{b(i), b(j)} & \text { if } i=1 \text { and } j=n\end{array}\right.$ as desired.

Finally let us consider the relation $R_{3}$, which essentially comes from the relations in the dihedral group $\mathcal{D}_{2 n}$. Making use of Reamrk 5, Lemma 3.1 and (3.1)-(3.2), by direct calculations we have

$$
\begin{aligned}
\psi(a)^{n} & =i_{*}\left(a_{1, n}^{n}\right)=i_{*}\left(\left(\sigma_{n-1} \sigma_{n-2} \cdots \sigma_{1}^{2} \cdots \sigma_{n-2} \sigma_{n-1}\right)\left(\sigma_{n-2} \cdots \sigma_{1}^{2} \cdots \sigma_{n-2}\right) \cdots \sigma_{1}^{2}\right) \\
& =i_{*}\left(\left(s_{1, n} s_{2, n} \cdots s_{n-1, n}\right)\left(s_{1, n-1} s_{2, n-1} \cdots s_{n-2, n-1}\right) \cdots s_{1,2}\right) \\
& =s_{1, n} s_{n-1, n} s_{n-2, n-1} \cdots s_{1,2}
\end{aligned}
$$

and

$$
\begin{aligned}
\psi(b)^{2} & =e_{2, n}^{2} e_{3, n-1}^{2} \cdots e_{r(n), s(n)}^{2} \quad\left(\text { by Lemma } 3.1\left(4^{\circ}\right)\right) \\
& =s_{2, n} s_{3, n-1} \cdots s_{r(n), s(n)} \quad(\text { by }(\underline{3.1})) \\
& =\left\{\begin{array}{ll}
e & \text { if } n \text { is even } \\
s_{\frac{n+1}{2} \frac{n+3}{2}} & \text { if } n \text { is odd }
\end{array} \quad \text { (since } \overline{i j} \notin E\left(C_{n}\right) \text { with }|i-j|>1 \text { except } \overline{1 n} \in E\left(C_{n}\right)\right) .
\end{aligned}
$$

On $\psi(b) \psi(a) \psi(b) \psi(a)$, by direct calculations we have that when $n=4$

$$
\begin{aligned}
\psi(b) \psi(a) \psi(b) \psi(a) & =i_{*}\left(\left(a_{2,4} a_{3,4}^{-1} a_{1,4}\right)^{2}\right)=\left(a_{2,4} a_{1,3}\right)^{2}=\left(\sigma_{3} \sigma_{2} \sigma_{2} \sigma_{1}\right)^{2}=\sigma_{3}^{2} \sigma_{1}^{2} \\
& \left.=s_{1,2} s_{3,4} \text { (since } \overline{24} \notin E\left(C_{4}\right)\right)
\end{aligned}
$$

and when $n=5$,

$$
\begin{aligned}
\psi(b) \psi(a) \psi(b) \psi(a) & =i_{*}\left(\left(a_{2,5} a_{3,5}^{-1} a_{3,4} a_{1,5}\right)^{2}\right)=\left(a_{2,5} a_{3,5}^{-1} a_{1,5} a_{4,5}\right)^{2} \\
& =\left(a_{2,5} a_{1,3} a_{4,5}\right)^{2}=\left(\sigma_{4} \sigma_{3} \sigma_{1} \sigma_{4}\right)^{2}=\sigma_{4}^{2} \sigma_{3}^{2} \sigma_{1}^{2} \\
& =s_{1,2} s_{3,4} s_{4,5}\left(\text { since } \overline{35} \notin E\left(C_{5}\right)\right) .
\end{aligned}
$$


More generally, when $n \geq 6$, by Lemma 3.1 we have that

$$
\begin{aligned}
\psi(b) \psi(a) \psi(b) \psi(a) & =i_{*}\left(e_{2, n} e_{3, n-1} \cdots e_{r(n), s(n)} a_{1, n}\right)^{2}=i_{*}\left(e_{2, n} a_{1, n} e_{4, n} \cdots e_{r(n)+1, s(n)+1}\right)^{2} \\
& =i_{*}\left(a_{2, n} a_{3, n}^{-1} a_{1, n} e_{4, n} \cdots e_{r(n)+1, s(n)+1}\right)^{2}=i_{*}\left(a_{3, n} \sigma_{2}^{2} \sigma_{1} e_{4, n} \cdots e_{r(n)+1, s(n)+1}\right)^{2} \\
& =i_{*}\left(a_{3, n} e_{4, n} \cdots e_{r(n)+1, s(n)+1}\right)^{2} \sigma_{1}^{2}=i_{*}\left(a_{3, n} e_{4, n} \cdots e_{r(n)+1, s(n)+1}\right)^{2} s_{1,2} .
\end{aligned}
$$

We see that $i_{*}\left(a_{3, n} e_{4, n} \cdots e_{r(n)+1, s(n)+1}\right)^{2}$ is only revelent to the path

$$
\Gamma^{\prime}: 3-4-\cdots-n
$$

in $C_{n}$, which can be regarded as the path $1-2-\cdots-(n-2)$ of $C_{n-2}$ on vertex set $[n-2]=\{1,2, \ldots, n-2\}$. Furthermore, in a similar way to the proof of Lemma 2.2, we may obtain that the inclusion $j: F\left(\mathbb{C}, C_{n-2}\right) \longrightarrow F\left(\mathbb{C}, \Gamma^{\prime}\right)$ induces an epimorhpism $j_{*}:\left.B\left(C_{n-2}\right)\right|_{\operatorname{Aut}\left(\Gamma^{\prime}\right)} \longrightarrow B\left(\Gamma^{\prime}\right)$. Thus

$$
i_{*}\left(a_{3, n} e_{4, n} \cdots e_{r(n)+1, s(n)+1}\right)^{2}
$$

becomes

$$
j_{*}\left(i_{*}\left(a_{1, n-2} e_{2, n-2} \cdots e_{r(n-2)+1, s(n-2)+1}\right)^{2}\right),
$$

in which $i_{*}\left(a_{1, n-2} e_{2, n-2} \cdots e_{r(n-2)+1, s(n-2)+1}\right)^{2}$ is exactly $(\psi(a) \psi(b))^{2}$ in $B\left(C_{n-2}\right)$. This means that we can get the expression of $\psi(b) \psi(a) \psi(b) \psi(a)$ by induction.

We have proved the cases of $n=4,5$. Now assume inductively that

$$
\psi(b) \psi(a) \psi(b) \psi(a)= \begin{cases}s_{1,2} s_{k+1, k+2} & \text { if } n=2 k \\ s_{1,2} s_{k+1, k+2} s_{k+2, k+3} & \text { if } n=2 k+1 .\end{cases}
$$

Then, as discussed above,

$$
\begin{aligned}
i_{*}\left(a_{1, n} e_{2, n} \cdots e_{r(n)+1, s(n)+1}\right)^{2} & =(\psi(a) \psi(b))^{2}=\psi(b)^{-1} \psi(b) \psi(a) \psi(b) \psi(a) \psi(b) \\
& = \begin{cases}\psi(b)^{-1} s_{1,2} s_{k+1, k+2} \psi(b) & \text { if } n=2 k \\
\psi(b)^{-1} s_{1,2} s_{k+1, k+2} s_{k+2, k+3} \psi(b) & \text { if } n=2 k+1\end{cases} \\
& = \begin{cases}s_{1,2 k} s_{k, k+1} & \text { if } n=2 k \\
s_{1,2 k+1} s_{k, k+1} s_{k+1, k+2} & \text { if } n=2 k+1 .\end{cases}
\end{aligned}
$$

When $n=2 k+2$,

$$
\psi(b) \psi(a) \psi(b) \psi(a)=j_{*}\left(s_{1,2 k} s_{k, k+1}\right) \sigma_{1}^{2}=i_{*}\left(s_{3,2 k+2} s_{k+2, k+3}\right) \sigma_{1}^{2}=s_{1,2} s_{k+2, k+3}
$$

in $B\left(C_{2 k+2}\right)$ and when $n=2 k+3$,

$$
\begin{aligned}
\psi(b) \psi(a) \psi(b) \psi(a) & =j_{*}\left(s_{1,2 k+1} s_{k, k+1} s_{k+1, k+2}\right) \sigma_{1}^{2}=i_{*}\left(s_{3,2 k+3} s_{k+2, k+3} s_{k+3, k+4}\right) \sigma_{1}^{2} \\
& =s_{1,2} s_{k+2, k+3} s_{k+3, k+4}
\end{aligned}
$$

in $B\left(C_{2 k+3}\right)$, as desired. This completes the proof.

\section{REFERENCES}

[1] Artin E. Theorie der Zöpfe. Ahb. Math. Sem. Univ. Hamburg, 1925, 6: 47-72.

[2] Artin E. Theory of braids. Ann. of Math, 1947, 48: 101-126.

[3] Arnol'd V I. On some topological invariants of algebrac functions. Trudy Moskov. Mat. Obshch, 1970, 21: 27-46; English transi. Trans. Moscow Math. Soc. 1970, 21: 30-52.

[4] Arnol'd V I. The cohomology ring of the colored braid group. Mat. Zametki, 1969, 5: 227-231; English transi. Math. Notes, 1969, 5: 138-1402. 
[5] Beineke L W, Wilson R J, Cameron P J. Topics in algebraic graph theory. Vol. 102. Cambridge University Press, 2004.

[6] Berrick A J, Cohen F R, Wong Y L, Wu J. Configurations, braids, and homotopy groups. J. Amer. Math. Soc, 2006, 19(2): 265-326.

[7] Birman J S. Braids, links, and mapping class groups. Annals of Mathematics Studies, Vol. 82. Princeton Univ. Press, Princeton, NJ 1974.

[8] Brieskorn E. Die Fundamentalgruppe des Raumes der regulären Orbits einer endlichen komplexen Spiegelungsgruppe. Invent. Math, 1971, 12: 57-61.

[9] Brieskorn E. Sur les groupes de tresses (d'après V.I. Arnol'd). Séminaire Bourbaki, 24ème année 1971/1972. Lecture Notes in Math, 1973, 317: 21-44.

[10] Brieskorn E, Saito K. Artin-Gruppen und Coxeter-Gruppen. Invent. Math, 1972, 17: 245-271.

[11] Deligne P. Les immeubles des groupes de tresses généralisés. Invent. Math, 1972, 17: 273-302.

[12] Eastwood M, Huggett S. Euler characteristics and chromatic polynomials. European J. Combin, 2007, 28(6): 1553-1560.

[13] Fox R, Neuwirth L. The braid groups. Math. Scand, 1962, 10: 119-126.

[14] Jones V F R. Hecke algebra representations of braid groups and link polynomials. Ann. of Math, 1987, 126: 335-388.

[15] Li H, Lü Z, Li F L. A theory of orbit braids. arXiv preprint:1903.11501v2, 2019.

[16] Looijenga E. Artin groups and the fundamental groups of some moduli spaces. J. Topol, 2008, 1(1): 187-216.

[17] Markoff A. Foundations of the algebraic theory of tresses. Trudy Matematicheskogo Instituta imeni VA Steklova, 1945, 16: 3-53.

[18] Parshin A N, Collins D J, Grigorchuk R I, et al. Algebra VII: Combinatorial Group Theory: Applications to Geometry[M]. Vol 7. Springer Science and Business Media, 1993.

[19] Randell R. The fundamental group of the complement of a union of complexhyperplanes. Invent. Math, 1982, 69(1): 103-108.

[20] Randell R. The fundamental group of the complement of a union of complex hyperplanes: correction. Invent. math, 1985, 80: 467-468.

[21] Rhodes F. On the fundamental group of a transformation group. Proceedings of the London Mathematical Society, 1966, 3(1): 635-650.

School of Mathematical Sciences, Fudan University, Shanghai, 200433, P. R. China.

E-mail address: $14110840001 @$ fudan.edu.cn

School of Mathematical Sciences, Fudan University, Shanghai, 200433, P. R. China.

E-mail address: zluefudan.edu.cn 